Article

Subscriber access provided by King Abdullah University of Science and Technology Library

\title{
A Self-Templating Scheme for the Synthesis of Nanostructured Transition Metal Chalcogenide Electrodes for Capacitive Energy Storage
}

\author{
Chuan Xia, and Husam N. Alshareef
}

Chem. Mater., Just Accepted Manuscript • DOI: 10.1021/acs.chemmater.5b01128 • Publication Date (Web): 11 Jun 2015

Downloaded from http://pubs.acs.org on June 14, 2015

\section{Just Accepted}

"Just Accepted" manuscripts have been peer-reviewed and accepted for publication. They are posted online prior to technical editing, formatting for publication and author proofing. The American Chemical Society provides "Just Accepted" as a free service to the research community to expedite the dissemination of scientific material as soon as possible after acceptance. "Just Accepted" manuscripts appear in full in PDF format accompanied by an HTML abstract. "Just Accepted" manuscripts have been fully peer reviewed, but should not be considered the official version of record. They are accessible to all readers and citable by the Digital Object Identifier (DOI®). "Just Accepted" is an optional service offered to authors. Therefore, the "Just Accepted" Web site may not include all articles that will be published in the journal. After a manuscript is technically edited and formatted, it will be removed from the "Just Accepted" Web site and published as an ASAP article. Note that technical editing may introduce minor changes to the manuscript text and/or graphics which could affect content, and all legal disclaimers and ethical guidelines that apply to the journal pertain. ACS cannot be held responsible for errors or consequences arising from the use of information contained in these "Just Accepted" manuscripts. 


\title{
A Self-Templating Scheme for the Synthesis of Nanostructured Transition Metal Chalcogenide Electrodes for Capacitive Energy Storage
}

\author{
Chuan Xia, Husam N. Alshareef*
}

\author{
C. Xia, Prof. H. N. Alshareef \\ Material Science and Engineering, King Abdullah University of Science and Technology \\ (KAUST), Thuwal 23955-6900, Saudi Arabia \\ *E-mail: husam.alshareef@kaust.edu.sa
}

\begin{abstract}
Due to their unique structural features including well-defined interior voids, low density, low coefficients of thermal expansion, large surface area and surface permeability, hollow micro/nanostructured transition metal sulfides with high conductivity have been investigated as new class of electrode materials for pseudocapacitor applications. Herein, we report a novel self-templating strategy to fabricate well-defined single and double-shell $\mathrm{NiCo}_{2} \mathrm{~S}_{4}$ hollow spheres, as a promising electrode material for pseudocapacitors. The surfaces of the $\mathrm{NiCo}_{2} \mathrm{~S}_{4}$ hollow spheres consist of self-assembled 2D mesoporous nanosheets. This unique morphology results in a high specific capacitance (1263 $\mathrm{F} \mathrm{g}^{-1}$ at $2 \mathrm{~A} \mathrm{~g}^{-1}$ ), remarkable rate performance $\left(75.3 \%\right.$ retention of initial capacitance from $2 \mathrm{~A} \mathrm{~g}^{-1}$ to $\left.60 \mathrm{~A} \mathrm{~g}^{-1}\right)$ and exceptional reversibility with a cycling efficiency of $93.8 \%$ and $87 \%$ after 10,000 and 20,000 cycles, respectively, at a high current density of $10 \mathrm{~A} \mathrm{~g}^{-1}$. The cycling stability of our ternary chalcogenides is comparable to carbonaceous electrode materials, but with much higher specific capacitance (higher than any previously reported ternary chalcogenide), suggesting that these unique chalcogenide structures have potential application in next-generation commercial pseudocapacitors.
\end{abstract}




\section{Introduction}

The fact that fossil fuels will ultimately be depleted has increased efforts aimed at using renewable energy sources in our daily life. Yet, the intermittency and unstable nature of some renewable energy sources such as solar and wind energy has driven the demand for energy storage devices. ${ }^{1-4}$ Batteries, which are expected to feature prominently in any renewable energy system, suffer from low power density and short cycling stability, ${ }^{1,5,6}$ which can limit their utilization. In this case, supercapacitors, which offer a much longer life cycle, higher power density and safer operation than batteries, can be used to compliment batteries, where high power is needed. Supercapacitors can be generally classified into two types, depending on the underlying energy storage mechanism. One type is the electrical double-layer capacitors (EDLCs) which store electrical energy by charge accumulation at the electrode/electrolyte interface. The other type is pseudocapacitors which are based on the fast and reversible redox reactions at the surface of electro-active materials, thus offering much higher specific capacitance and energy density than EDLCs. ${ }^{1,7-9}$ Previously developed pseudocapacitors have been based on transition metal oxides, hydroxides and conducting polymers which invariably suffer from either low conductivity or poor electrochemical stability, largely limiting their applications. ${ }^{10-14}$ Therefore, it is imperative to create alternative pseudocapacitive materials with low cost, desirable electrical conductivity, highly porous structure, high specific surface area, desirable ionic permeability, large capacitance and good electrochemical stability. ${ }^{15,16}$

Due to their unique structural features including well-defined interior voids, low density, low coefficients of thermal expansion, large surface area and surface permeability, hollow micro/nanostructured binary transition metal sulfides such as nickel sulfide and cobalt sulfide 
have been widely investigated as a new class of pseudocapacitive electrode materials. ${ }^{17-22}$ Unfortunately, the previous reports showed either relatively low specific capacitance or poor rate capability, as well as quite limited enhancement of cycle life. In addition, the post-removal of templates in the template-based methods always required an additional time-consuming step to remove the template.

Very recently, it has been reported that mixed transition-metal-sulfides particularly nickel cobalt sulfides $\left(\mathrm{NiCo}_{2} \mathrm{~S}_{4}\right)$ showed excellent properties when used as pseudocapacitive material. These results are due to the intrinsic stability, higher electrical conductivity $(\sim 100$ times as high as that of $\mathrm{NiCo}_{2} \mathrm{O}_{4}$ and much higher than those of the binary sulfides), and richer redox reactions than the corresponding single component sulphides, in much the same way as $\mathrm{NiCo}_{2} \mathrm{O}_{4}$ to the corresponding single component oxides. ${ }^{23-26}$ In addition, it has been well demonstrated that an outer protection layer is important to stabilize the nanostructured ultrathin hollow spheres. ${ }^{27-29}$ In this sense, double-shell hollow structures of $\mathrm{NiCo}_{2} \mathrm{O}_{4}$ with protection outer layer, large surface area, highly porous structure and desirable ionic permeability may be an excellent potential pseudocapacitive material. To date, while binary transition-metal-sulfides have been well established, it remains a challenge to fabricate well-defined hollow structures of ternary transition-metal-sulfides such as $\mathrm{NiCo}_{2} \mathrm{~S}_{4}$.

In this paper, we report a self-templating strategy to controllably fabricate well-defined $\mathrm{NiCo}_{2} \mathrm{~S}_{4}$ (NCS) single and double-shell hollow spheres (HS) for high performance pseudocapacitors. The size of the interior void of these hollow spheres could be easily tuned by tailoring the size of the starting silica template, generating significant difference in electrochemical performance. Even more, the size effect of template and morphology-dependent enhancement of pseudocapacitive performance is demonstrated. 


\section{Experimental section}

2.1 Synthesis of silica template. Monodisperse $\mathrm{SiO}_{2}$ solid spheres with varied size were prepared using a modified Stober precess. ${ }^{30}$ In a typical synthesis of $\sim 750 \mathrm{~nm}$ silica, $6 \mathrm{~mL}$ of TEOS was rapidly added into a mixture of $62 \mathrm{~mL}$ of ethanol and $12 \mathrm{~mL}$ of ammonia (30-30\%). The mixture was stirred mildly for $1 \mathrm{~h}$, repeatedly washed with DI water and ethanol. To acquire $\sim 490 \mathrm{~nm}$ and $\sim 380 \mathrm{~nm}$ silica, typically, $9 \mathrm{~mL}$ or $3 \mathrm{~mL}$ of ammonia (30-33\%) as catalyst was added into the starting reagents contained $4.5 \mathrm{~mL}$ of TEOS, $24.75 \mathrm{~mL}$ of $\mathrm{H}_{2} \mathrm{O}$, and 61.75 or $67.75 \mathrm{~mL}$ of ethanol, respectively. Afterwards, this mixture was kept at room temperature for $2 \mathrm{~h}$, and followed washing and drying. Particularly, $\sim 100 \mathrm{~nm}$ silica was obtained from a mixture of $2 \mathrm{~mL}$ of TEOS, $8 \mathrm{~mL}$ of ammonia (30-33\%), $75 \mathrm{~mL}$ of methanol and $10 \mathrm{~mL}$ of DI water. After stirring at room temperature for $2 \mathrm{~h}$, the products were washed and dried in air.

2.2 Synthesis of Ni-Co hydrosilicate precursor. Typically, $5 \mathrm{mmol}$ of silica solid sphere template with different diameter (Supplemental Figure S16) or commercial silica nanoparticle were dispersed in $40 \mathrm{~mL}$ of DI water, followed by the addition of $16.7 \mathrm{mmol}$ of urea, $0.3 \mathrm{mmol}$ of $\mathrm{Ni}\left(\mathrm{NO}_{3}\right)_{2} \cdot 6 \mathrm{H}_{2} \mathrm{O}$, and $0.6 \mathrm{mmol}$ of $\mathrm{Co}\left(\mathrm{NO}_{3}\right)_{2} \cdot 6 \mathrm{H}_{2} \mathrm{O}$. After vigorous stirring at room temperature for $0.5 \mathrm{~h}$, the thoroughly mixed solution was kept at $105{ }^{\circ} \mathrm{C}$ for $12 \mathrm{~h}$ under mildly stirring. After cooling down naturally, the product was centrifuged and washed.

2.3 Synthesis of $\mathrm{NiCo}_{2} \mathrm{~S}_{4}$ single-shell hollow spheres and $\mathrm{NiCo}_{2} \mathrm{~S}_{4}$ nanosheets. $0.1 \mathrm{~g}$ of the asprepared precursor was re-dispersed into $40 \mathrm{~mL}$ of DI water, following by the addition of $0.3 \mathrm{~g}$ of $\mathrm{Na}_{2} \mathrm{~S}$. After vigorous stirring for $15 \mathrm{~min}$, this solution was subsequently transferred into a Teflon-lined autoclave and kept at $160^{\circ} \mathrm{C}$ for $12 \mathrm{~h}$. The final product was washed and dried in a 
vacuum at $60^{\circ} \mathrm{C}$. After drying, the as-prepared powder was annealed under $\mathrm{N}_{2}$ atmosphere at 300 degree for $0.5 \mathrm{~h}$ prior to $\mathrm{x}$-ray diffraction measurement to determine the crystal phase.

2.4 Synthesis of $\mathrm{NiCo}_{2} \mathrm{~S}_{4}$ double-shell hollow spheres. The core-shell NCS@ $\mathrm{SiO}_{2}$ structure were prepared through a versatile Stober sol-gel methods as follow. ${ }^{30,31} 60 \mathrm{mg}$ of NCS HS was redispersed into $280 \mathrm{~mL}$ of ethanol, followed by the addition of $70 \mathrm{~mL}$ of DI water and $5 \mathrm{~mL}$ of ammonia (30-33\%). Afterwards, $4 \mathrm{~mL}$ of TEOS was added by dropwise in $10 \mathrm{~min}$, and the reaction was kept at room temperature for $12 \mathrm{~h}$ under mildly stirring to obtain the $\mathrm{NCS} @ \mathrm{SiO}_{2}$ core-shell products. Next, $0.1 \mathrm{~g}$ of the resultant core-shell structure was added into $40 \mathrm{~mL}$ of DI water which contained $0.3 \mathrm{~g} \mathrm{Na}_{2} \mathrm{~S}$. After thoroughly mixed, this solution was kept at $160{ }^{\circ} \mathrm{C}$ for $12 \mathrm{~h}$ in the autoclave. After centrifugation and wash, the resultant was annealed under $\mathrm{N}_{2}$ atmosphere at 300 degree for $0.5 \mathrm{~h}$ prior to $\mathrm{x}$-ray diffraction measurement to determine the crystal phase.

2.5 Materials characterization. The morphology and microstructure were investigated by SEM (Nova Nano 630, FEI) and TEM (Titan 80-300 kV (ST) TEM, FEI), and the elemental presence and composition were identified using energy-dispersive X-ray spectroscopy (EDS) and XPS analysis (Kratos AXIS Ultra DLD). The XRD measurement was conducted on a powder X-ray diffraction system (XRD, Bruker, D8 ADVANCE) equipped with $\mathrm{Cu} \mathrm{K} \alpha$ radiation $(\lambda=0.15406$ $\mathrm{nm})$. BET surface area of the samples were determined using surface area and porosimetry system "Micromeritics" (ASAP 2420) at $77 \mathrm{~K}$. Before measurements, the samples were dried at $70{ }^{\circ} \mathrm{C}$ for $10 \mathrm{~h}$ in a Vacuum oven and then degassed at $200^{\circ} \mathrm{C}$ for $6 \mathrm{~h}$ until the vacuum was less than $2 \mu \mathrm{m} \mathrm{Hg}$.

2.6 Electrochemical measurements. The electrochemical tests were carried out at room temperature in three-electrode (half-cell) configurations with $\mathrm{NiCo}_{2} \mathrm{~S}_{4}$ as a working electrode, a 
Pt wire as the counter electrode, and saturated calomel electrode (SCE) as the reference electrode. The working electrodes with a mass loading of $\sim 1 \mathrm{mg} \mathrm{cm}^{-2}$ were prepared by coating the active $\mathrm{NiCo}_{2} \mathrm{~S}_{4}$, acetylene black and polyvinylidene fluride (PVDF) in a weight ratio of $7: 2: 1$ on the carbon cloth. $1 \mathrm{M} \mathrm{KOH}$ was used as electrolyte for all measurements.

The specific capacitance was then calculated from the galvanostatic charge-discharge (CD) curves as:

$$
C=\frac{I}{m \frac{\Delta V}{\Delta t}}
$$

Where $m$ is the total mass loading of the electrode, $I$ is the constant current for charge-discharge and $\frac{\Delta V}{\Delta t}$ is slope of the discharge curve. ${ }^{32}$

\section{Results and discussion}

As schematically illustrated in Figure 1A, the synthesis strategy of $\mathrm{NiCo}_{2} \mathrm{~S}_{4}$ hollow spheres can be generally described as hydrolysis-sulfidation process. First, as-prepared $350 \mathrm{~nm}$ silica spheres were used as a template and raw material to prepare nickel cobalt silicate hydroxide precursor through a facile hydrolysis process at $105{ }^{\circ} \mathrm{C}$ (see Methods section for details). As shown in Supplementary Figure S1, the color of starting materials changes from light gray to pink after the hydrolysis process, demonstrating the formation of nickel cobalt hydrosilicate, which originates from the co-precipitation of $\mathrm{Ni}^{2+}$ and $\mathrm{Co}^{2+}$ cations on the surface of monodispersed silica nanospheres in an alkaline solution. ${ }^{33}$ In order to investigate the phase and structure at various stages of the reaction, X-ray diffraction (XRD) patterns were recorded. The typical XRD pattern of the precursor (Supplementary Figure S2) showed one broad diffraction peak around $23^{\circ}$, which can be assigned to the amorphous silica phase. In addition, the two peaks around $34^{\circ}$ and $60^{\circ}$ could be assigned to the (200) and (600) planes of crystalline 
$(\mathrm{Ni}, \mathrm{Co})_{3} \mathrm{Si}_{2} \mathrm{O}_{5}(\mathrm{OH})_{4}$ phase, although the exact crystal structure remains unsolved. ${ }^{34}$ The asprepared precursor retains the original morphology of the silica template with a diameter of 300 $\mathrm{nm}$, as clearly shown in Figure 1B and Supplementary Figure S3. The corresponding high-angle annular dark-field scanning transmission electron microscopy (HAADF-STEM) image and elemental analysis also reveal the core-shell structure and the successful formation of $\mathrm{Ni}-\mathrm{Co}$ hydrosilicate of our $(\mathrm{Ni}, \mathrm{Co})_{3} \mathrm{Si}_{2} \mathrm{O}_{5}(\mathrm{OH})_{4} @ \mathrm{SiO}_{2}$ precursor (Supplementary Figure S4). The obvious empty gap between the inner silica core and the outer Ni-Co hydrosilicate shell shows that the silica not only serves as a template, but also gets involved in the hydrolysis reaction. Next, the well-defined $\mathrm{NiCO}_{2} \mathrm{~S}_{4}$ hollow spheres (HS) were derived from the precursor via a facile in-situ sulfidation process using $\mathrm{Na}_{2} \mathrm{~S}$ as sulfur source. During the chemical transformation process, the silica cores are etched simultaneously by $\mathrm{NaOH}$, which is released from the hydrolysis of $\mathrm{Na}_{2} \mathrm{~S}$, eliminating the need for an additional etching step to remove the silica template. The crystal phase and structure of the product after sulfidation were investigated by XRD. All diffraction peaks (Supplementary Figure S2) can be assigned to thiospinel $\mathrm{NiCo}_{2} \mathrm{~S}_{4}$ without any noticeable second phases.

Figure $1 \mathrm{C}-\mathrm{D}$ reveals the morphology and microstructure of the as-obtained $\mathrm{NiCo}_{2} \mathrm{~S}_{4}$ hollow spheres (Black solution in Supplementary Figure S1). Interestingly, these hierarchical hollow spheres are entirely self-assembled from ultrathin mesoporous $\mathrm{NiCo}_{2} \mathrm{~S}_{4}$ nanosheets with a thickness of few nanometers (Supplementary Figure S5), ${ }^{20}$ thereby resulting in large surface-tovolume ratio and highly mesoporous structure. Such a structure offers a large interfacial area between the electroactive $\mathrm{NiCo}_{2} \mathrm{~S}_{4}$ and the electrolyte ions, and short diffusion paths for fast ionic diffusion, which is highly desirable for pseudocapacitors. From the close-up STEM images in Supplementary Figure S6, an outer rough surface of the hollow spheres, consisting of curved 
nanosheets, with a thickness around $20 \mathrm{~nm}$, can be discerned. The lattice fringes with different orientations shown in Figure $1 \mathrm{~F}$ suggest that the as-obtained $\mathrm{NiCo}_{2} \mathrm{~S}_{4} \mathrm{HS}$ is polycrystalline in nature, which is confirmed by the corresponding selected-area electron-diffraction (SAED) pattern in Figure $1 \mathrm{G}$.

Energy-dispersive X-ray spectroscopy (EDS) and X-ray photoelectron spectroscopy (XPS) were carried out to provide further insight into the hollow interior as well as the chemical composition and oxidation states of the as-prepared $\mathrm{NiCo}_{2} \mathrm{~S}_{4} \mathrm{HS}$. Figure $1 \mathrm{E}$ shows the EDSSTEM line scans of the $300 \mathrm{~nm} \mathrm{NiCo} 2 \mathrm{~S}_{4} \mathrm{HS}$ for nickel, cobalt and sulfur. The profiles of Ni, Co and $\mathrm{S}$ have a broad spectrum throughout the entire diameter but are more prominent at the boundary region, corresponding to the hollow nature of the of $\mathrm{NiCo}_{2} \mathrm{~S}_{4}$ spheres. Figure 2 shows the typical energy-filtered transmission electron microscopy (EFTEM) elemental mapping for $300 \mathrm{~nm} \mathrm{NiCo} 2 \mathrm{~S}_{4}$ HS. A uniform distribution of the elements $\mathrm{Ni}, \mathrm{Co}$, and $\mathrm{S}$ on the surface of the hollow sphere is clearly visible, further demonstrating the successful preparation of thiospinel NiCo2S4. High-resolution XPS spectra of Co $2 p$, Ni $2 p$ and S $2 p$ core levels have been recorded and are shown in Supplementary Figure S7, which matches well with those obtained for ternary $\mathrm{NiCo}_{2} \mathrm{~S}_{4} \cdot{ }^{23-25}$ From the survey scans of XPS spectrum (Supplementary Figure S7A), the presence of small amount of $\mathrm{Si}$ is likely to be the amorphous silicate residue. ${ }^{20}$ Furthermore, $\mathrm{N}_{2}$ adsorption/desorption measurements (Supplementary Figure S8) demonstrate that the $\mathrm{NiCo}_{2} \mathrm{~S}_{4}$ HS themselves are mesoporous in nature with uniform pores of around $3 \mathrm{~nm}$, possessing a large BET surface area of $122 \mathrm{~m}^{2} \mathrm{~g}^{-1}$. Such highly mesoporous HS structure with exceptional surface permeability for electrolyte ions is the ideal morphology for pseudocapacitor applications because it can enhance electrochemical surface reactions. 
It has been reported that the sphere size can significantly affect the electrochemical performance for nanostructured hollow spheres in other material systems. ${ }^{35,36}$ We have therefore prepared various sizes of $\mathrm{NiCo}_{2} \mathrm{~S}_{4}$ hollow spheres $(700 \mathrm{~nm}, 450 \mathrm{~nm}, 300 \mathrm{~nm}$ and $100 \mathrm{~nm}$ in diameter) using different size silica templates (see Method for details), henceforth referred to as 700nm-NCS to $100 \mathrm{~nm}$-NCS. The electrochemical properties of different size $\mathrm{NiCo}_{2} \mathrm{~S}_{4}$ HSs were investigated by three-electrode configuration in $1.0 \mathrm{M} \mathrm{KOH}$ solution. Figure 3A-B shows the cyclic voltammetry (CV) and galvanostatic discharge curves obtained at $20 \mathrm{mV} \mathrm{s}^{-1}$ and $10 \mathrm{~A} \mathrm{~g}^{-1}$ for all samples, respectively. One pronounced pair of peaks can be observed in the representative $\mathrm{CV}$ curves, clearly revealing the pseudocapacitive characteristics of these materials. ${ }^{25,37,38}$ The formation of the broad redox peaks can be attributed to the highly reversible Faradaic reactions of $\mathrm{Ni}^{2+} / \mathrm{Ni}^{3+}$ and $\mathrm{Co}^{2+} / \mathrm{Co}^{3+} / \mathrm{Co}^{4+}$ pairs with electrolyte anions $\mathrm{OH}^{-}$, according to the following equation: $:^{26,39}$

$$
\begin{gathered}
\mathrm{NiCO}_{2} \mathrm{~S}_{4}+2 \mathrm{OH}^{-} \leftrightarrow \mathrm{NiS}_{4-2 x} \mathrm{OH}+2 \mathrm{CoS}_{x} \mathrm{OH}+2 e^{-} \\
\mathrm{CoS}_{x} \mathrm{OH}+\mathrm{OH}^{-} \leftrightarrow \mathrm{CoS}_{x} \mathrm{O}+\mathrm{H}_{2} \mathrm{O}+e^{-}
\end{gathered}
$$

Moreover, multiple distinct voltage plateaus are manifested in the galvanostatic discharge profiles which suggest the presence of reversible redox reactions, consistent with the electrochemical behavior of the $\mathrm{CV}$ curves. The small voltage (IR) drop observed in the galvanostatic discharge curves is attributed to the high conductivity of $\mathrm{NiCo}_{2} \mathrm{~S}_{4},{ }^{23}$ resulting in low equivalent series resistances (ESR) which results in high pseudocapacitive performance. At the same scan rate of $20 \mathrm{mV} \mathrm{s}^{-1}$, it is obvious that the integrated area of CV curves (from 700nmNCS to $100 \mathrm{~nm}-\mathrm{NCS}$ ) firstly increases and then decreases, suggesting a strong influence of the sphere size on electrode capacitance. The galvanostatic discharge curves also illustrate varied discharge periods at current density of $10 \mathrm{~A} \mathrm{~g}^{-1}$, matching with the results from $\mathrm{CV}$ 
measurements. Figure 3C presents the specific capacitance for all studied samples. It is clear that 300nm-NCS HS has higher specific capacitance (997 $\mathrm{F} \mathrm{g}^{-1}$ at $2 \mathrm{~A} \mathrm{~g}^{-1}$ ) than the other samples (901, 929 and $745 \mathrm{~F} \mathrm{~g}^{-1}$ for 700nm-NCS, 450nm-NCS and 100nm-NCS at $2 \mathrm{~A} \mathrm{~g}^{-1}$, respectively). Figure $3 \mathrm{D}$ clearly shows that the pseudocapacitive performance of our $\mathrm{NiCo}_{2} \mathrm{~S}_{4}$ hollow spheres is strongly correlated to the diameter size, especially for the cycling stability performance (Figure 3E); similar phenomena of size-dependent electrochemical performance was also observed in battery materials such as $\mathrm{SnO}_{2}$ hollow spheres. ${ }^{35}$ The sphere size effect on the $\mathrm{NiCo}_{2} \mathrm{~S}_{4}$ performance can be explained by the following factors. First, the BET surface area of the four studied hollow sphere sizes initially increases from $700 \mathrm{~nm}-\mathrm{NCS}$ to $300 \mathrm{~nm}-\mathrm{NCS}$ and then decrease dramatically from $300 \mathrm{~nm}-\mathrm{NCS}$ to $100 \mathrm{~nm}-\mathrm{NCS}$, a fact that has been revealed by the BET measurement (Supplementary Figure S8). The larger surface area can offer a higher concentration of electrochemically active sites, larger interfacial area between the electroactive materials and the electrolyte ions, and shorter diffusion paths for fast ionic diffusion, all of which can promote the electrochemical performance. ${ }^{40}$ Second, it is well known that the spherical shell geometry is capable of withstanding extreme stresses, and the maximum tensile stress in a hollow $\mathrm{Si}$ sphere is $\sim 5$ times lower than that of a solid $\mathrm{Si}$ sphere of equal volume, during electrochemical reaction. ${ }^{35,41,42}$ Therefore, the larger size $\mathrm{NiCo}_{2} \mathrm{~S}_{4}$ HS is more fragile during the long-term cycling measurement. Hence, the smaller sized silica template results in better $\mathrm{NiCo}_{2} \mathrm{~S}_{4} \mathrm{HS}$ integrity which can endure the large volume change without pulverization. ${ }^{35}$ Third, as mentioned above, the silica in this case is used as a starting material and plays the role of a self-dissolving template. The empty gap between shells shown in Figure 1B successfully demonstrates the partial dissolution of silica template as it participates in the hydrolysis reaction. As illustrated in Figure 3F, and Supplementary Figure S9, the 700, 450 and $300 \mathrm{~nm}$ silica 
spheres are large enough to act as template, resulting in a uniform morphology and well-defined $\mathrm{NiCo}_{2} \mathrm{~S}_{4} \mathrm{HS}$. Yet, the data appears to show that the $100 \mathrm{~nm}$ silica template might be too small to support the formation of an outer shell. Thus, the morphology of final product prepared by templating with $100 \mathrm{~nm}$ silica (Figure 3F and Supplementary Figure S10) is a mixture of random quasi-hollow spheres and nanosheets with the lowest BET surface area among all samples. These factors result in the worst electrochemical performance and worst capacitance retention for the 100nm-NCS. This particular morphology is full of defects and cracks, which can more easily lead to pulverization of the $\mathrm{NiCo}_{2} \mathrm{~S}_{4}$ electrode into small fragments. Thus, we can conclude that there is a critical sphere size for obtaining good electrochemical performance for our $\mathrm{NiCo}_{2} \mathrm{~S}_{4}$ HS electrodes. The optimized 300nm-NCS HS electrode exhibits a high specific capacitance of $997 \mathrm{~F} \mathrm{~g}^{-1}$ at $2 \mathrm{~A} \mathrm{~g}^{-1}$, good rate performance and impressive long cycle life at high current density of $10 \mathrm{~A} \mathrm{~g}^{-1}$ (retention $90.3 \%$ and $82 \%$ after 10,000 and 20,000 cycles, respectively). This result is one of the most stable pseudocapacitive materials among the reported materials in the literature.

As we have demonstrated in the previous section, the $\mathrm{NiCo}_{2} \mathrm{~S}_{4}$ hollow sphere electrodes made using $300 \mathrm{~nm}$ silica sacrificial template display optimal electrochemical performance. Notwithstanding the remarkable performance, the cycle life of $300 \mathrm{~nm}-\mathrm{NCS}$ HS need to be competitive with commercial carbonaceous material electrodes. Recognizing, that an outer protection layer can be used to stabilize hollow electrode structures, we decided to study the effect of placing a second chalcogenide shell around the first one. Hence, a second highly conductive $\mathrm{NiCo}_{2} \mathrm{~S}_{4}$ protection shell was directly coated on the first $300 \mathrm{~nm}-\mathrm{NCS}$ hollow sphere, resulting in a $\mathrm{NiCo}_{2} \mathrm{~S}_{4}$ double shell structure. As illustrated in Figure 4A, a TEOS (tetraethyl orthosilicate)-driven silica was used to deposit a uniform and conformally deposited layer on the surface of $300 \mathrm{~nm}-\mathrm{NCS}$ hollow spheres via a versatile Stober sol-gel method. ${ }^{27,31}$ Afterwards, the 
$\mathrm{NiCo}_{2} \mathrm{~S}_{4}$ outer shell was formed using the same hydrolysis-sulfidation process (see Method section for details). Figure 4B-C reveals TEM images with different magnification for 300nmNCS HS@SiO 2 core-shell structure. Obviously, the NCS HS retains the $300 \mathrm{~nm}$ hollow interior with around $100 \mathrm{~nm}$ second shell of silica. After the same hydrolysis-sulfidation process, the dense silica outer shell transforms into highly porous nanosheet arrays on the surface, with a thickness of around $150 \mathrm{~nm}$, as shown in Figure 4D-E and Supplementary Figure S11. XRD measurement shows again single $\mathrm{NiCo}_{2} \mathrm{~S}_{4}$ phase for the $\mathrm{NCS}$ double shell structure (Supplementary Figure S12). To demonstrate the morphology-dependent improvement of electrochemical properties, the results of $\mathrm{NiCo}_{2} \mathrm{~S}_{4}$ nanosheets (Supplementary Figure $\mathrm{S} 13$ ) preparation based on commercial silica nanoparticle is included for comparison (see Method for details). The $\mathrm{N}_{2}$ adsorption/desorption experiments were used to measure the surface area of single and double shell hollow spheres, which had a BET surface area of 29,122 , and $117 \mathrm{~m}^{2} \mathrm{~g}^{-1}$, respectively. Figure 5A-B shows the $\mathrm{CV}$ and $\mathrm{CD}$ profiles of NCS double-shell hollow spheres under different scan rate and current density. They manifest similar redox peaks and distinct voltage plateaus as the single-shell NCS hollow spheres, suggesting the analogous pseudocapacitive behavior. The CD profiles show highly symmetric feature of the chargedischarge process, implying the high efficiency of $\mathrm{NiCo}_{2} \mathrm{~S}_{4}$ electrode, excellent reversibility of redox reactions and their superior rate capability as well. The anodic peaks shift to higher potential while the cathodic peaks shift to lower potential with increased scan rate due to the fast charge and discharge rates. ${ }^{25}$ This yields wider potential difference between anodic and cathodic peaks, resulting in a capacitance decrease. Supplemental Figure S14 demonstrates the linear relationship of anodic and cathodic peaks presented in Figure 5A versus the square root of scan rate, indicating the dominance of diffusion-controlled electrode reactions, which is one of the 
characteristics of pseudocapacitive materials. ${ }^{43-45}$ Figure $5 \mathrm{C}$ shows $\mathrm{CV}$ curves of single and double-shell NCS hollow spheres measured at the same scan rate of $20 \mathrm{mV} \mathrm{s}^{-1}$. It can be clearly seen that the integrated area under the CV curve is higher for the double-shell NCS hollow spheres, meaning better electrochemical performance. Furthermore, the longer discharge period observed in Figure 5D of NCS double-shell hollow sphere confirms their better performance, a fact that is consistent with the results observed in the $\mathrm{CV}$ measurements. Figure 5E-F summarizes the detailed pseudocapacitive performance for the three products with different morphology. It is evident that the double-shell NCS hollow spheres show the highest specific capacitance of $1263 \mathrm{~F} \mathrm{~g}^{-1}$ at $2 \mathrm{~A} \mathrm{~g}^{-1}$, remarkable rate capability $(75.3 \%$ retention of initial capacitance from $2 \mathrm{~A} \mathrm{~g}^{-1}$ to $\left.60 \mathrm{~A} \mathrm{~g}^{-1}\right)$, as well as superior cycling stability $(93.8 \%$ and $87 \%$ capacitance retention after 10,000 and 20,000 cycles at a high current density of $10 \mathrm{~A} \mathrm{~g}^{-1}$, respectively). These results are superior to those of the single-shell NCS hollow sphere and the NCS nanosheet. Based on the above impressive performance, especially the super-long cycle life which is comparable to that of carbonaceous materials, we can conclude that the $\mathrm{NiCo}_{2} \mathrm{~S}_{4}$ with this unique structure is a promising materials for next-generation pseudocapacitors.

To sum up, the NCS double-shell hollow spheres exhibit the best performance among the different $\mathrm{NiCo}_{2} \mathrm{~S}_{4}$ morphologies studied in this work. Our performance is superior to that of all reported ternary nickel cobalt sulfides based materials (literature reports were summarized in Supplementary Table S1). ${ }^{24,34,38,40,46-50}$ This phenomenon may be attributed to the unique double shell structure which offers many desirable features. First of all, the highly porous double shells surfaces are covered with self-assembled nanosheets which are full of mesopores (with size distribution centered around 3-5 nm, Supplemental Figure S7B). These can provide significant number of electroactive sites and shorter diffusion paths for charge transports, facilitating the 
redox reactions compared to their bulk or solid counterparts. ${ }^{20}$ Besides, the nanometer-sized sheets are able to reduce diffusion time of the electrolyte ions, and can accommodate the strain during the $\mathrm{OH}^{-}$insertion and extraction during electrochemical cycling. ${ }^{51}$ Further, the sacrificialtemplate growth process that we have developed results in robust adhesion between the inner and outer shells, leading to better stability during cycling. In addition, the NCS double shell structure offers an impressively high surface area, suggesting the possibility of the exposure of numerous active sites available for reaction on the electrode surface. ${ }^{40}$ Moreover, the higher meso/macropore volume of double-shell NCS can boost the electrolyte ions trapping and access to the active sites. In addition, the vertically coated curved nanosheet arrays of the NCS double-shells can increase the contact area between neighboring double-shells (Supplementary Figure S15) and provide a superb pathway for fast electron transportation through the electrodes. ${ }^{25,52}$ Thus, we observed a higher electrical conductivity of NCS double-shells $(720 \mu \Omega \mathrm{cm})$ over its single shell counterparts $(1600 \mu \Omega \mathrm{cm})$. What's more, the void space in the hollow structure can better accommodate any volume change during cycling ${ }^{53}$ and the thicker shell of the double-shell structure (150 nm vs. $20 \mathrm{~nm}$ for single shell $300 \mathrm{~nm}-\mathrm{NCS}$ ) with high conductivity can more efficiently endure the extremely long cycling measurements, leading to better cycling performance as shown in Figure 5d (89\% capacitance retention of NCS double-shells vs. $82 \%$ retention of $300 \mathrm{~nm}-\mathrm{NCS}$ single shell after 20,000 cycles). In contrast, the much poorer mechanical integratity of monodispersed or aggregated NCS nanosheets makes them pulverize more easily during electrochemical cycling, presenting unsatisfactory cycling stability (only 34\% capacitance retention after 20,000 cycles). These results demonstrate that such the spherical double shell construction of $\mathrm{NiCO}_{2} \mathrm{~S}_{4}$ produces a pseudocapacitive material with superb performance. 


\title{
4. Conclusion
}

In summary, we have demonstrated a facile hydrolysis-sulfidation strategy to fabricate well-defined $\mathrm{NiCo}_{2} \mathrm{~S}_{4}$ hollow spheres with single and double shell configuration. The unique attributes of the double shell hollow spheres include stable structure, high surface area, high permeability and void space, and remarkable pseudocapacitive performance in terms of ratability and extreme cycling stability. The cycling stability of our ternary chalcogenides is comparable to carbonaceous electrode materials, but with much higher specific capacitance (higher than any previously reported ternary chalcogenide), suggesting that these unique $\mathrm{NiCo}_{2} \mathrm{~S}_{4}$ electrodes are promising pseudocapacitive materials for commercial applications. Importantly, this work also provides new insight into the controllable fabrication of mixed transition metal chalcogenides with excellent properties.

\author{
ASSOCIATED CONTENT \\ Supporting Information. XRD, SEM, TEM, HAADF-STEM, XPS, EDS analysis, and BET measurements for \\ precursor, single and double shell $\mathrm{NiCo}_{2} \mathrm{~S}_{4}$ are included as Supporting Information. This material is available free of \\ charge via the Internet at http://pubs.acs.org.
}

\section{AUTHOR INFORMATION}

\section{Corresponding Author}

*Email: husam.alshareef@kaust.edu.sa

\section{Acknowledgements}

Research reported in this publication has been supported by King Abdullah University of Science and Technology (KAUST). C.X. thanks Dr. Peng Li, Dr. Rakhi, Dr. Narendra and Bilal Ahemed at KAUST for several useful 
discussions. The authors wish to thank the staff of the Imaging and Characterization Laboratory at KAUST, especially Dr. Nini Wei and Dr. Chao Zhao for their help with the TEM analysis and Dr. Nejib Hedhili for his help with the XPS measurements. 


\section{Reference}

(1) Simon, P.; Gogotsi, Y. Materials for electrochemical capacitors. Nat Mater 2008, 7, 845-854.

(2) Wang, K.; Wu, H. P.; Meng, Y. N.; Wei, Z. X. Conducting Polymer Nanowire Arrays for High Performance Supercapacitors. Small 2014, 10, 14-31.

(3) Xiong, P.; Liu, B.; Teran, V.; Zhao, Y.; Peng, L.; Wang, X.; Yu, G. Chemically Integrated Two-Dimensional Hybrid Zinc Manganate/Graphene Nanosheets with Enhanced Lithium Storage Capability. Acs Nano 2014, 8, 86108616.

(4) Xiong, P.; Peng, L.; Chen, D.; Zhao, Y.; Wang, X.; Yu, G. Two-dimensional nanosheets based Li-ion full batteries with high rate capability and flexibility. Nano Energy 2015, 12, 816-823.

(5) Armand, M.; Tarascon, J. M. Building better batteries. Nature 2008, 451, 652-657.

(6) Winter, M.; Brodd, R. J. What are batteries, fuel cells, and supercapacitors? Chem Rev 2005, 105, 1021-1021.

(7) Inagaki, M.; Konno, H.; Tanaike, O. Carbon materials for electrochemical capacitors. J Power Sources 2010, 195, 7880-7903.

(8) Snook, G. A.; Kao, P.; Best, A. S. Conducting-polymer-based supercapacitor devices and electrodes. J Power Sources 2011, 196, 1-12.

(9) Zhang, L. L.; Zhao, X. S. Carbon-based materials as supercapacitor electrodes. Chem Soc Rev 2009, 38, 25202531.

(10) Rakhi, R. B.; Chen, W.; Cha, D.; Alshareef, H. N. Nanostructured Ternary Electrodes for Energy-Storage Applications. Adv Energy Mater 2012, 2, 381-389.

(11) Jiang, F. R.; Li, W. Y.; Zou, R. J.; Liu, Q.; Xu, K. B.; An, L.; Hu, J. Q. MoO3/PANI coaxial heterostructure nanobelts by in situ polymerization for high performance supercapacitors. Nano Energy 2014, 7, 72-79.

(12) Chen, W.; Xia, C.; Rakhi, R. B.; Alshareerf, H. N. A general approach toward enhancement of pseudocapacitive performance of conducting polymers by redox-active electrolytes. J Power Sources 2014, 267, 521-526.

(13) Zhang, X.; Zeng, X. Z.; Yang, M.; Qi, Y. X. Investigation of a Branchlike MoO3/Polypyrrole Hybrid with Enhanced Electrochemical Performance Used as an Electrode in Supercapacitors. Acs Appl Mater Inter 2014, 6, $1125-1130$.

(14) Dong, X. L.; Guo, Z. Y.; Song, Y. F.; Hou, M. Y.; Wang, J. Q.; Wang, Y. G.; Xia, Y. Y. Flexible and Wire-

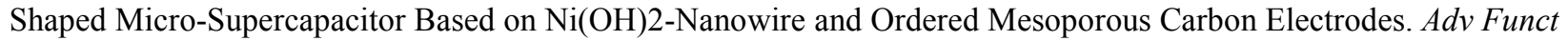
Mater 2014, 24, 3405-3412.

(15) Wang, G. P.; Zhang, L.; Zhang, J. J. A review of electrode materials for electrochemical supercapacitors. Chem Soc Rev 2012, 41, 797-828.

(16) Yan, J.; Wang, Q.; Wei, T.; Fan, Z. J. Recent Advances in Design and Fabrication of Electrochemical Supercapacitors with High Energy Densities. Adv Energy Mater 2014, 4, 1300816.

(17) Wan, H. Z.; Ji, X.; Jiang, J. J.; Yu, J. W.; Miao, L.; Zhang, L.; Bie, S. W.; Chen, H. C.; Ruan, Y. J. Hydrothermal synthesis of cobalt sulfide nanotubes: The size control and its application in supercapacitors. $J$ Power Sources 2013, 243, 396-402.

(18) Apostolova, R. D.; Shembel, E. M.; Talyosef, I.; Grinblat, J.; Markovsky, B.; Aurbach, D. Study of electrolytic

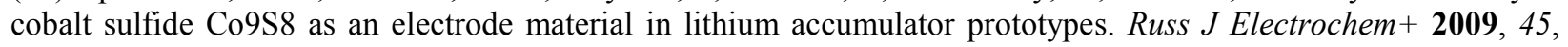
311-319.

(19) Peng, S. J.; Li, L. L.; Tan, H. T.; Cai, R.; Shi, W. H.; Li, C. C.; Mhaisalkar, S. G.; Srinivasan, M.; Ramakrishna, S.; Yan, Q. Y. MS 2 ( $\mathrm{M}=\mathrm{Co}$ and Ni) Hollow Spheres with Tunable Interiors for High- Performance Supercapacitors and Photovoltaics. Adv Funct Mater 2014, 24, 2155-2162.

(20) Zhu, T.; Wang, Z. Y.; Ding, S. J.; Chen, J. S.; Lou, X. W. Hierarchical nickel sulfide hollow spheres for high performance supercapacitors. Rsc Adv 2011, 1, 397-400.

(21) Yang, Z. S.; Chen, C. Y.; Chang, H. T. Supercapacitors incorporating hollow cobalt sulfide hexagonal nanosheets. J Power Sources 2011, 196, 7874-7877.

(22) Shen, L.; Yu, L.; Wu, H. B.; Yu, X.-Y.; Zhang, X.; Lou, X. W. D. Formation of nickel cobalt sulfide ball-inball hollow spheres with enhanced electrochemical pseudocapacitive properties. Nat Commun 2015, 6 .

(23) Xiao, J. W.; Wan, L.; Yang, S. H.; Xiao, F.; Wang, S. Design Hierarchical Electrodes with Highly Conductive NiCo2S4 Nanotube Arrays Grown on Carbon Fiber Paper for High-Performance Pseudocapacitors. Nano Lett 2014, $14,831-838$. 
(24) Pu, J.; Cui, F. L.; Chu, S. B.; Wang, T. L.; Sheng, E. H.; Wang, Z. H. Preparation and Electrochemical Characterization of Hollow Hexagonal NiCo2S4 Nanoplates as Pseudocapacitor Materials. Acs Sustain Chem Eng 2014, 2, 809-815.

(25) Chen, W.; Xia, C.; Alshareef, H. N. One-Step Electrodeposited Nickel Cobalt Sulfide Nanosheet Arrays for High-Performance Asymmetric Supercapacitors. Acs Nano 2014, 8, 9531-9541.

(26) Chen, H. C.; Jiang, J. J.; Zhang, L.; Xia, D. D.; Zhao, Y. D.; Guo, D. Q.; Qi, T.; Wan, H. Z. In situ growth of $\mathrm{NiCo} 2 \mathrm{~S} 4$ nanotube arrays on Ni foam for supercapacitors: Maximizing utilization efficiency at high mass loading to achieve ultrahigh areal pseudocapacitance. J Power Sources 2014, 254, 249-257.

(27) Shao, M. F.; Ning, F. Y.; Zhao, Y. F.; Zhao, J. W.; Wei, M.; Evans, D. G.; Duan, X. Core-Shell Layered Double Hydroxide Microspheres with Tunable Interior Architecture for Supercapacitors. Chem Mater 2012, 24, 1192-1197.

(28) Lou, X. W.; Li, C. M.; Archer, L. A. Designed Synthesis of Coaxial SnO2@carbon Hollow Nanospheres for Highly Reversible Lithium Storage. Adv Mater 2009, 21, 2536-+.

(29) Xu, H. L.; Wang, W. Z. Template synthesis of multishelled Cu2O hollow spheres with a single-crystalline shell wall. Angew Chem Int Edit 2007, 46, 1489-1492.

(30) Stober, W.; Fink, A.; Bohn, E. Controlled Growth of Monodisperse Silica Spheres in Micron Size Range. $J$ Colloid Interf Sci 1968, 26, 62-\&.

(31) Li, W.; Deng, Y. H.; Wu, Z. X.; Qian, X. F.; Yang, J. P.; Wang, Y.; Gu, D.; Zhang, F.; Tu, B.; Zhao, D. Y. Hydrothermal Etching Assisted Crystallization: A Facile Route to Functional Yolk-Shell Titanate Microspheres with Ultrathin Nanosheets-Assembled Double Shells. J Am Chem Soc 2011, 133, 15830-15833.

(32) V, K.; Frackowiak, E.; Beguin, F. Determination of the specific capacitance of conducting polymer/nanotubes composite electrodes using different cell configurations. Electrochim Acta 2005, 50, 2499-2506.

(33) Jin, P.; Chen, Q. W.; Hao, L. Q.; Tian, R. F.; Zhang, L. X.; Wang, L. Synthesis and catalytic properties of nickel-silica composite hollow nanospheres. J Phys Chem B 2004, 108, 6311-6314.

(34) Yu, L.; Zhang, L.; Wu, H. B.; Lou, X. W. Formation of NixCo3-xS4 Hollow Nanoprisms with Enhanced Pseudocapacitive Properties. Angew Chem Int Edit 2014, 53, 3711-3714.

(35) Kim, W. S.; Hwa, Y.; Jeun, J. H.; Sohn, H. J.; Hong, S. H. Synthesis of SnO2 nano hollow spheres and their size effects in lithium ion battery anode application. J Power Sources 2013, 225, 108-112.

(36) Balaya, P. Size effects and nanostructured materials for energy applications. Energ Environ Sci 2008, 1, 645654.

(37) Chen, X. Y.; Zhu, H. L.; Chen, Y. C.; Shang, Y. Y.; Cao, A. Y.; Hu, L. B.; Rubloff, G. W. MWCNT/V2O5 Core/Shell Sponge for High Areal Capacity and Power Density Li-Ion Cathodes. Acs Nano 2012, 6, 7948-7955.

(38) Chen, H. C.; Jiang, J. J.; Zhang, L.; Wan, H. Z.; Qi, T.; Xia, D. D. Highly conductive NiCo2S4 urchin-like nanostructures for high-rate pseudocapacitors. Nanoscale 2013, 5, 8879-8883.

(39) Mei, L.; Yang, T.; Xu, C.; Zhang, M.; Chen, L. B.; Li, Q. H.; Wang, T. H. Hierarchical mushroom-like CoNi2S4 arrays as a novel electrode material for supercapacitors. Nano Energy 2014, 3, 36-45.

(40) Zhu, Y. R.; Wu, Z. B.; Jing, M. J.; Yang, X. M.; Song, W. X.; Ji, X. B. Mesoporous NiCo2S4 nanoparticles as high-performance electrode materials for supercapacitors. J Power Sources 2015, 273, 584-590.

(41) Shan, Z. W.; Adesso, G.; Cabot, A.; Sherburne, M. P.; Asif, S. A. S.; Warren, O. L.; Chrzan, D. C.; Minor, A. M.; Alivisatos, A. P. Ultrahigh stress and strain in hierarchically structured hollow nanoparticles. Nat Mater 2008, 7 , 947-952.

(42) Yao, Y.; McDowell, M. T.; Ryu, I.; Wu, H.; Liu, N. A.; Hu, L. B.; Nix, W. D.; Cui, Y. Interconnected Silicon Hollow Nanospheres for Lithium-Ion Battery Anodes with Long Cycle Life. Nano Lett 2011, 11, $2949-2954$.

(43) Luo, F. L.; Li, J.; Yuan, H. Y.; Xiao, D. Rapid synthesis of three-dimensional flower-like cobalt sulfide hierarchitectures by microwave assisted heating method for high-performance supercapacitors. Electrochim Acta 2014, 123, 183-189.

(44) Kim, H.; Park, K. Y.; Hong, J.; Kang, K. All-graphene-battery: bridging the gap between supercapacitors and lithium ion batteries. Sci Rep-Uk 2014, 4.

(45) Chen, K. F.; Song, S. Y.; Li, K. Y.; Xue, D. F. Water-soluble inorganic salts with ultrahigh specific capacitance: crystallization transformation investigation of CuCl2 electrodes. Crystengcomm 2013, 15, 10367-10373.

(46) Zhang, Y. F.; Ma, M. Z.; Yang, J.; Sun, C. C.; Su, H. Q.; Huang, W.; Dong, X. C. Shape-controlled synthesis of $\mathrm{NiCo} 2 \mathrm{~S} 4$ and their charge storage characteristics in supercapacitors. Nanoscale 2014, 6, 9824-9830.

(47) Yang, J. Q.; Guo, W.; Li, D.; Qin, Q.; Zhang, J.; Wei, C. Y.; Fan, H. M.; Wu, L. Y.; Zheng, W. J. Hierarchical porous NiCo2S4 hexagonal plates: Formation via chemical conversion and application in high performance supercapacitors. Electrochim Acta 2014, 144, 16-21. 
(48) Pu, J.; Wang, T. T.; Wang, H. Y.; Tong, Y.; Lu, C. C.; Kong, W.; Wang, Z. H. Direct Growth of NiCo2S4 Nanotube Arrays on Nickel Foam as High-Performance Binder-Free Electrodes for Supercapacitors. Chempluschem 2014, 79, 577-583.

(49) Wan, H. Z.; Jiang, J. J.; Yu, J. W.; Xu, K.; Miao, L.; Zhang, L.; Chen, H. C.; Ruan, Y. J. NiCo2S4 porous nanotubes synthesis via sacrificial templates: high-performance electrode materials of supercapacitors. Crystengcomm 2013, 15, 7649-7651.

(50) Peng, S. J.; Li, L. L.; Li, C. C.; Tan, H. T.; Cai, R.; Yu, H.; Mhaisalkar, S.; Srinivasan, M.; Ramakrishna, S.; Yan, Q. Y. In situ growth of NiCo2S4 nanosheets on graphene for high-performance supercapacitors. Chem Commun 2013, 49, 10178-10180.

(51) Wang, X.; Wu, X. L.; Guo, Y. G.; Zhong, Y. T.; Cao, X. Q.; Ma, Y.; Yao, J. N. Synthesis and Lithium Storage Properties of Co3O4 Nanosheet-Assembled Multishelled Hollow Spheres. Adv Funct Mater 2010, 20, 1680-1686.

(52) Li, H. B.; Yu, M. H.; Wang, F. X.; Liu, P.; Liang, Y.; Xiao, J.; Wang, C. X.; Tong, Y. X.; Yang, G. W. Amorphous nickel hydroxide nanospheres with ultrahigh capacitance and energy density as electrochemical pseudocapacitor materials. Nat Commun 2013, 4.

(53) Taberna, L.; Mitra, S.; Poizot, P.; Simon, P.; Tarascon, J. M. High rate capabilities Fe3O4-based Cu nanoarchitectured electrodes for lithium-ion battery applications. Nat Mater 2006, 5, 567-573. 

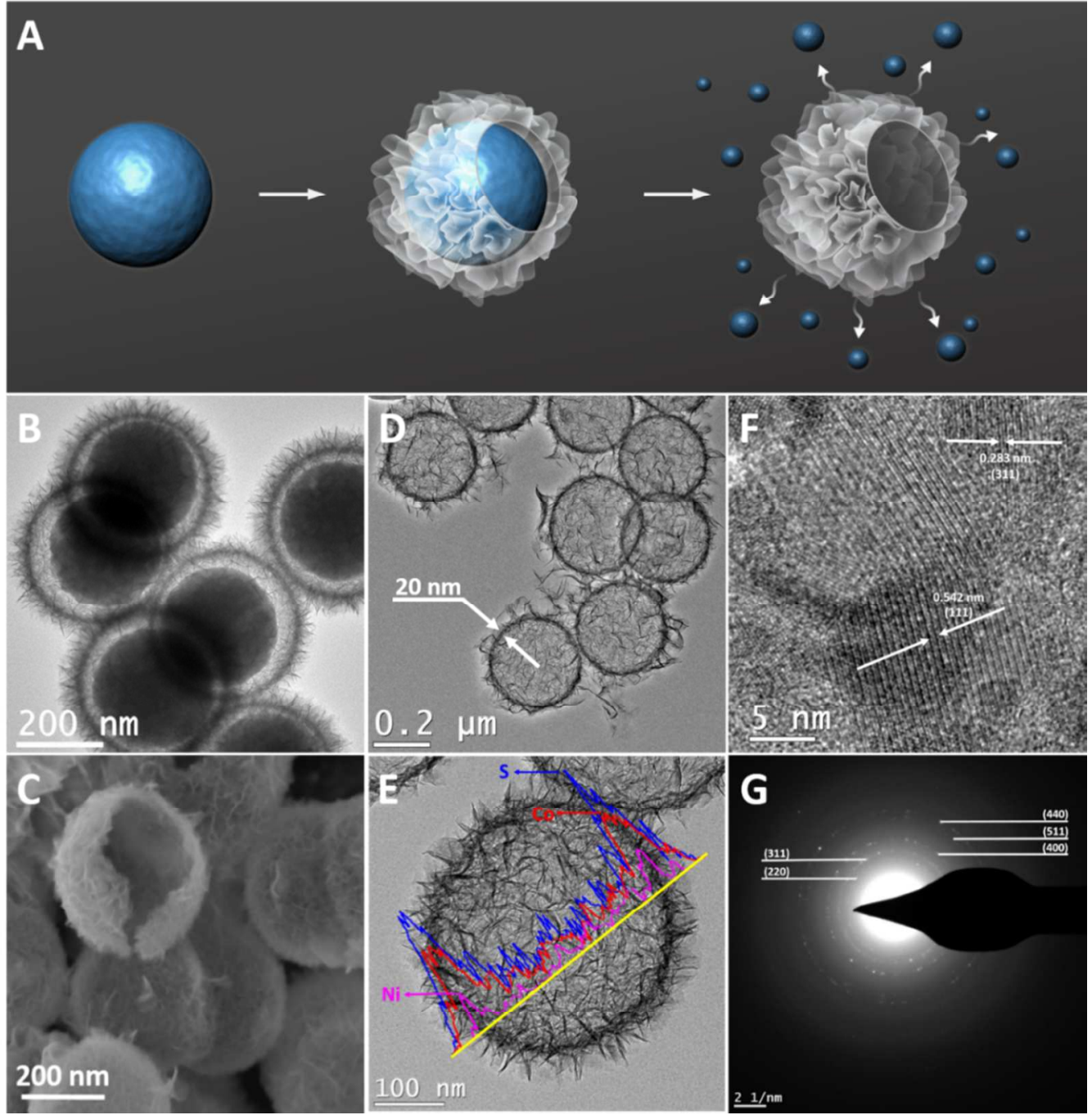

Figure 1. (A) Schematic of the synthesis of $\mathrm{NiCo}_{2} \mathrm{~S}_{4}$ hollow spheres. (B) TEM image of the 350 $\mathrm{nm}$ silica based precursor. (C) FESEM and (D) TEM images of $300 \mathrm{~nm} \mathrm{NiCo} 2 \mathrm{~S}_{4}$ hollow sphere obtained from Ni-Co hydrosilicate precursor. (E) EDS-STEM line scan of $300 \mathrm{~nm} \mathrm{NiCo} 2 \mathrm{~S}_{4}$ hollow sphere; the purple, blue and red lines represent counts of nickel, cobalt and sulfur signals along the solid yellow line, respectively. (F) HRTEM image and (G) the corresponding selectedarea electron-diffraction pattern of $300 \mathrm{~nm} \mathrm{NiCo}{ }_{2} \mathrm{~S}_{4}$ hollow sphere. 

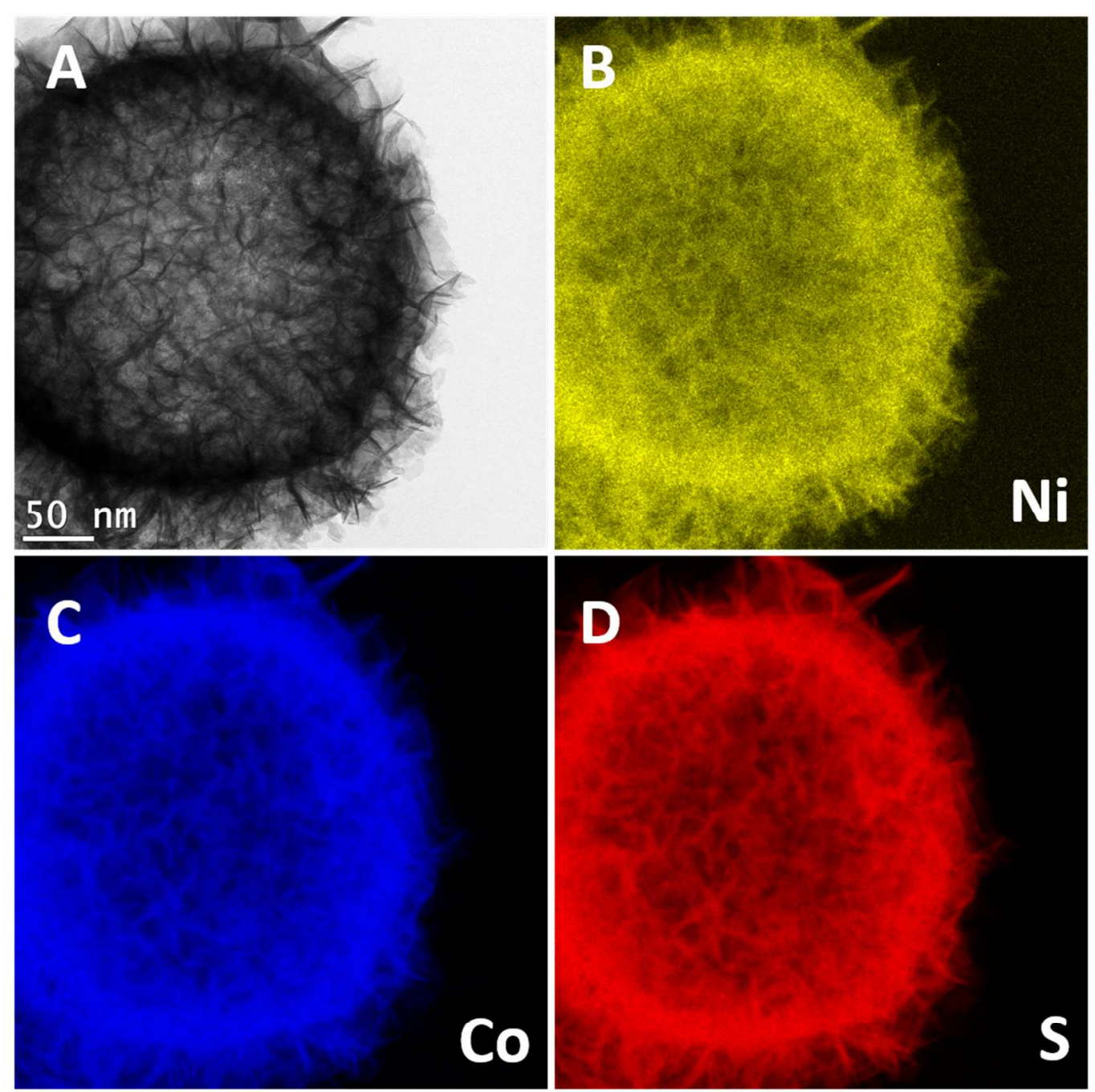

D

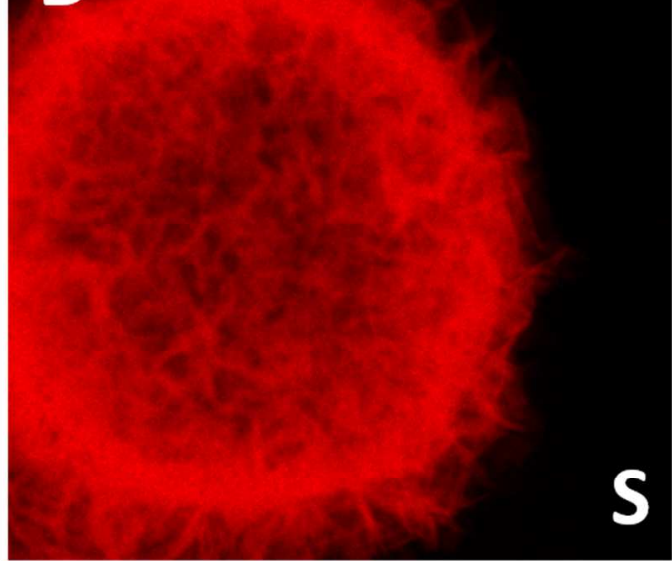

Figure 2. (A) Typical transmission secondary electron image of the $\mathrm{NiCo}_{2} \mathrm{~S}_{4}$ hollow sphere; and the corresponding energy-filtered transmission electron microscopy (EFTEM) elemental mapping of (B) nickel, (C) cobalt and (D) sulfur, respectively. 

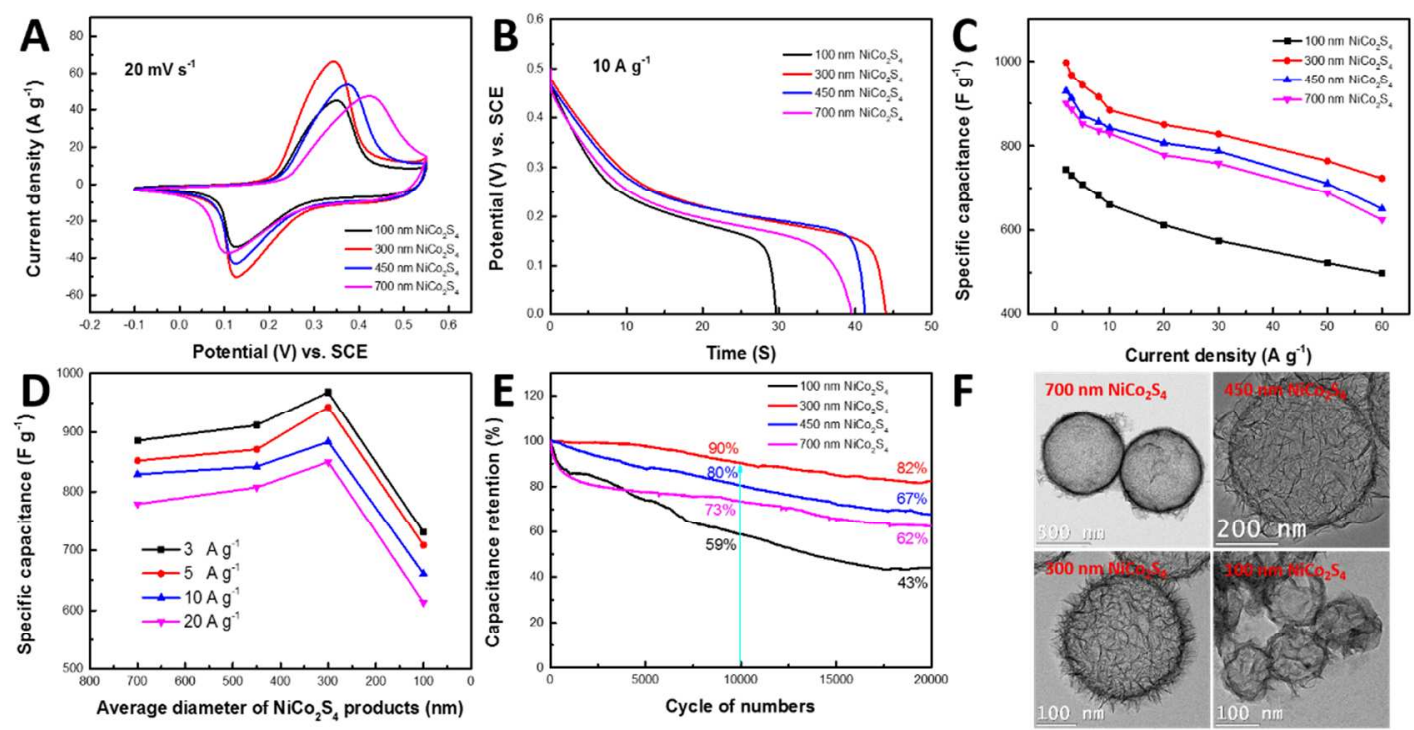

Figure 3. (A) Cyclic voltammetry curves at same scan rate of $20 \mathrm{mV} \mathrm{s}^{-1}$; (B) galvanostatic discharge profiles at same current density of $10 \mathrm{~A} \mathrm{~g}^{-1}$; and (C) summary of specific capacitance as a function of current density of all studied samples. (D) Summary of specific capacitance as a function of average diameter of $\mathrm{NiCo}_{2} \mathrm{~S}_{4}$ hollow sphere. (E) Cycling stability performance at current density of $10 \mathrm{~A} \mathrm{~g}^{-1}$ and (F) representative TEM images of all as-prepared samples. 

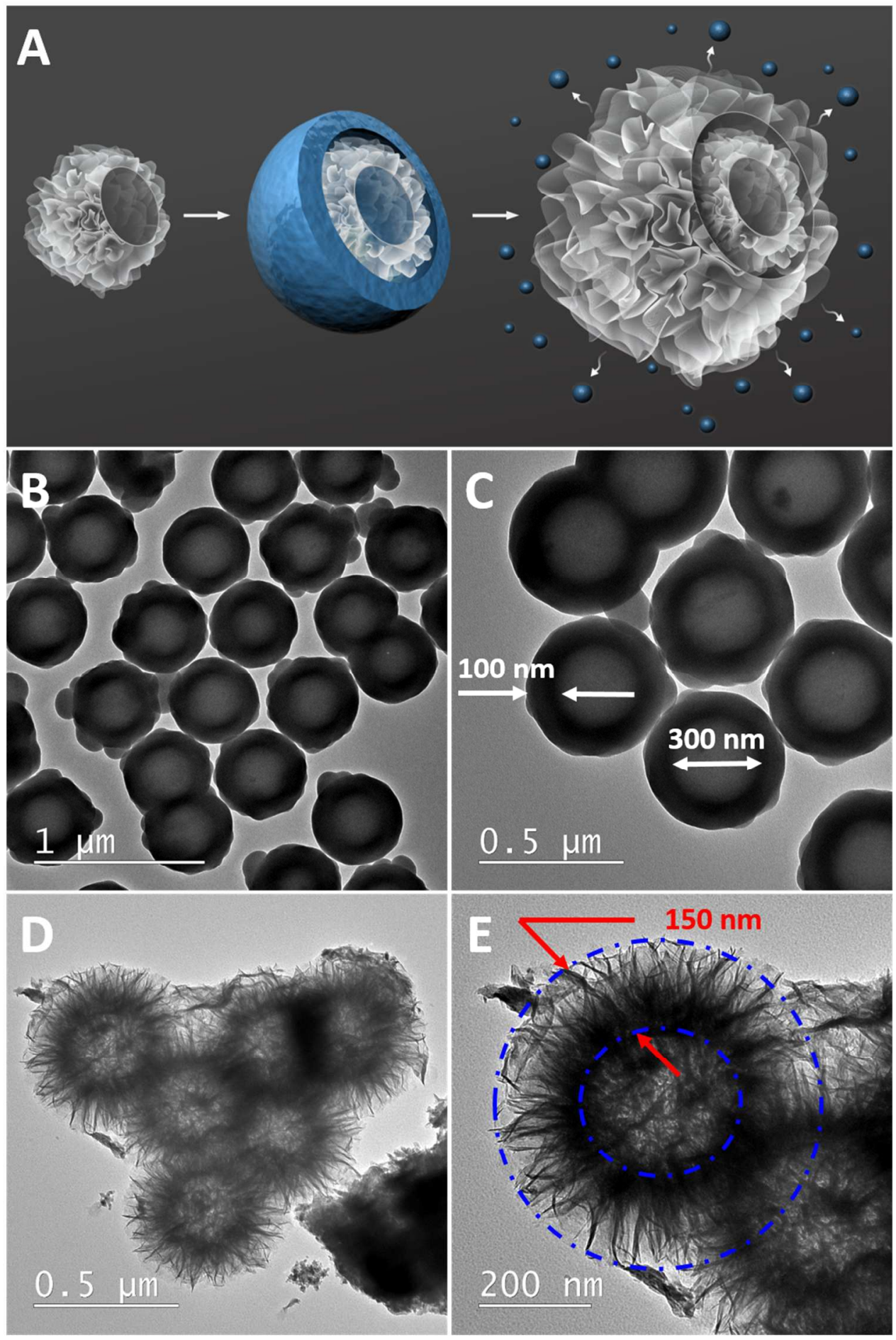

Figure 4. (A) Schematic illustration of the synthesis concept of $\mathrm{NiCo}_{2} \mathrm{~S}_{4}$ double shell hollow sphere. (B, C) TEM images of $300 \mathrm{~nm} \mathrm{NiCo} \mathrm{S}_{4}$ hollow sphere@ $\mathrm{SiO}_{2}$ core-shell structure. (D, E) TEM images of homogeneous $\mathrm{NiCo}_{2} \mathrm{~S}_{4}$ double shell hollow sphere with a shell thickness of around $150 \mathrm{~nm}$. 

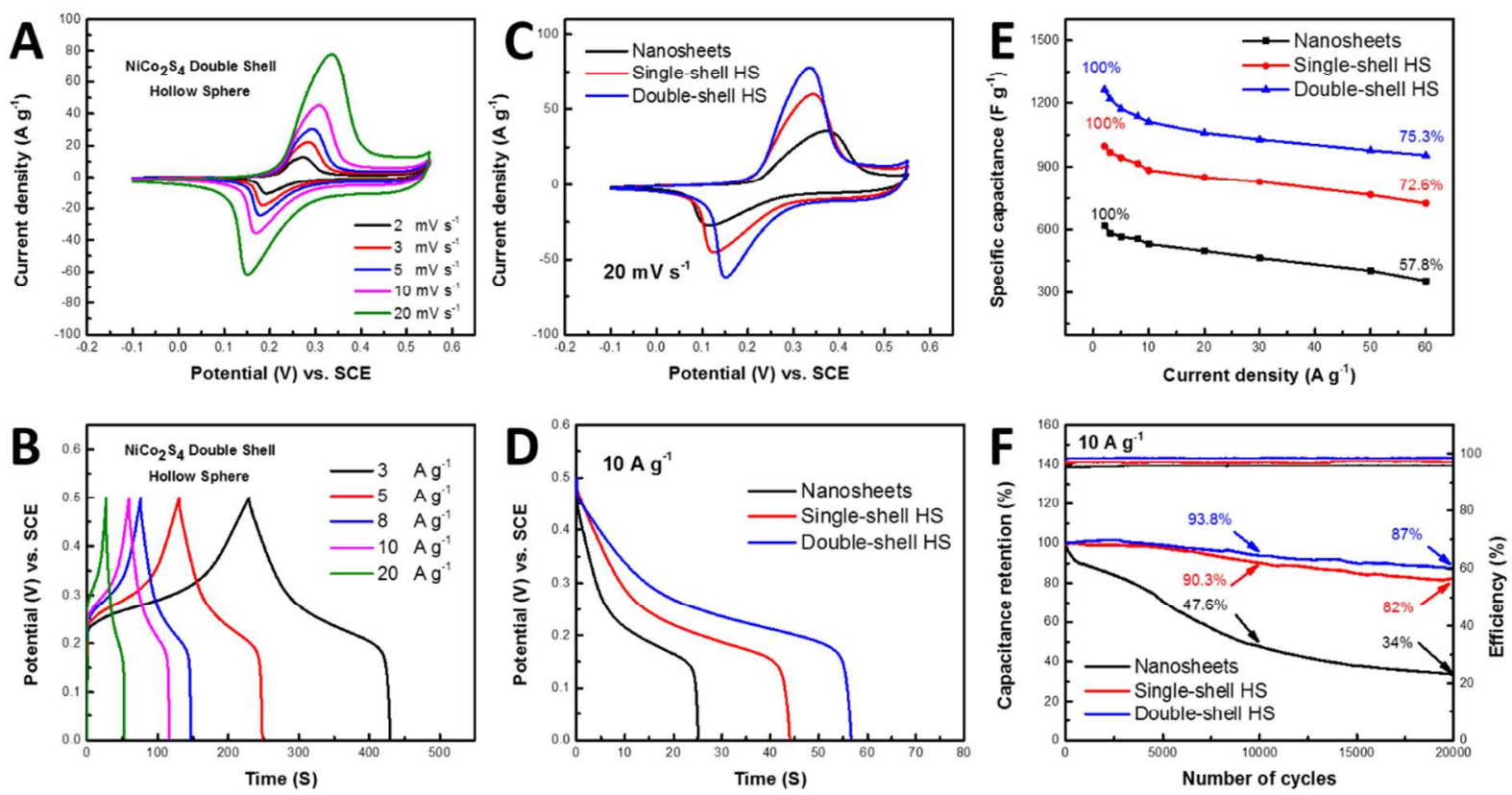

Figure 5. (A) Cyclic voltammetry and (B) galvanostatic charge-discharge profiles of $\mathrm{NiCo}_{2} \mathrm{~S}_{4}$ double shell hollow sphere (HS). (C) Comparison of cyclic voltammetry curves at same scan rate of $20 \mathrm{mV} \mathrm{s}^{-1}$ and (D) galvanostatic discharge profiles at same current density of $10 \mathrm{~A} \mathrm{~g}^{-1}$ of NCS nanosheets, single-shell HS and double-shell HS. (E) Summary of specific capacitance and (F) cycling stability performance at current density of $20 \mathrm{~A} \mathrm{~g}^{-1}$ of NCS nanosheets, single-shell HS and double-shell HS. 


\section{ToC}
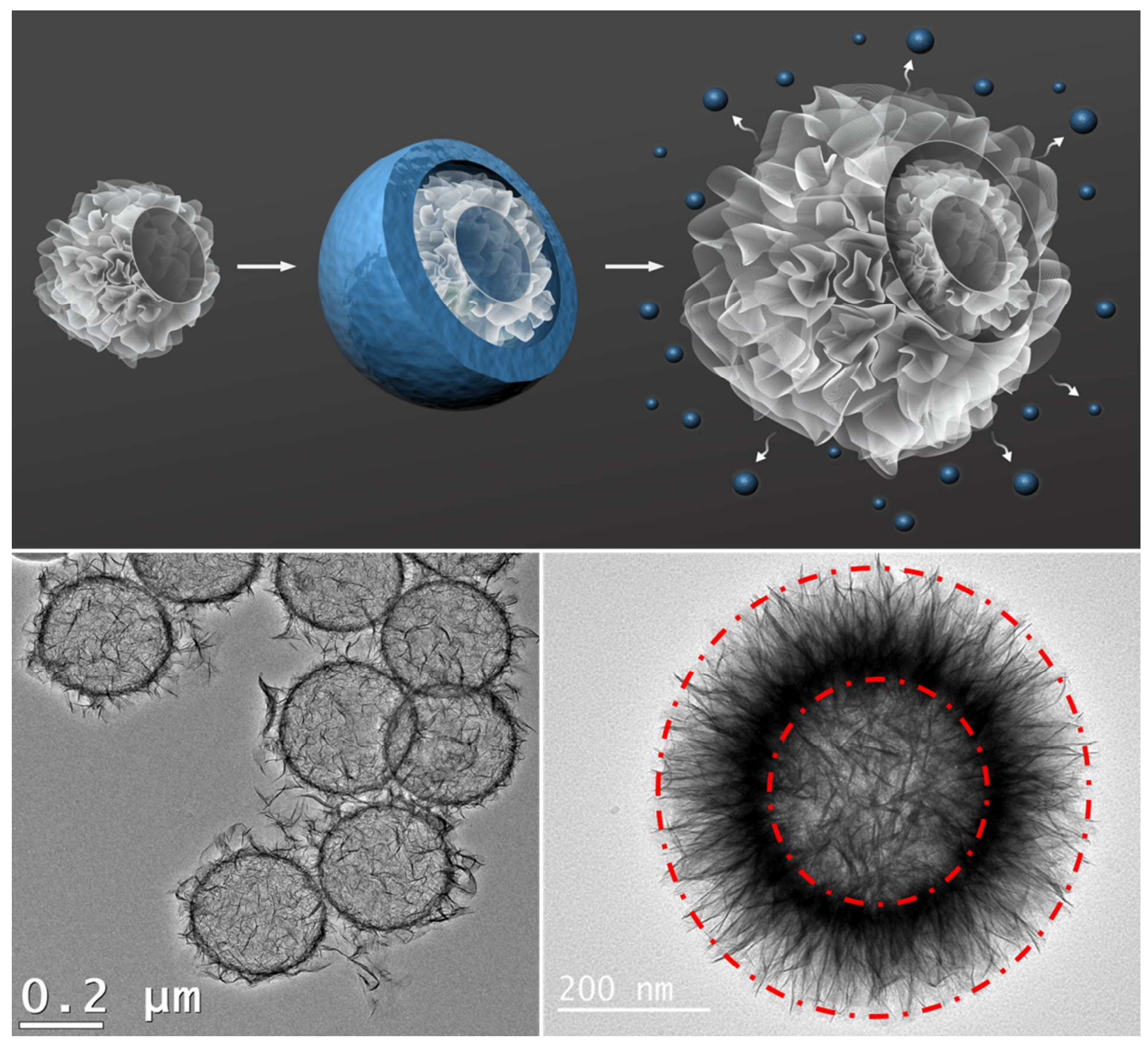